\title{
An Analysis of The Rationality of The Structure of Digitalize Troop's Information Network Based on Complex Network
}

\author{
Li Yipeng \\ Military College \\ the National University of Defense and Technology, \\ Changsha, China \\ Email: wingwing175@hotmail.com
}

\author{
Fu Guangming \\ Military College \\ the National University of Defense and Technology, \\ Changsha, China
}

\author{
Shen Yubo \\ Military College \\ the National University of Defense and Technology \\ Changsha, China
}

\begin{abstract}
To construct the information network of Digitalize Troops and analyze its efficiency, we must face the actual need of the battlefield, obey the standard of the capability of our arming; and while insuring the balance of network values structurally, actions must be taken to promote the comprehensive capability of the whole network. This article first expatiates on the structure of Digitalize Troop's information network, communication links, relative node units, and then use the knowledge from complex network to analyze the rationality of absolute link and downright flat structure of information network by setting plus-models of network value, and at the same time puts forward the relative index bound and requirements of the reasonable structure of network from a fix-quantify angle, thus providing references for the construction of the information network of the Digitalize Troops of our army macroscopically.
\end{abstract}

Keywords- Complex Network, Digitalize Troop's Information Network, the Rationality of the Structure

\section{INTRODUCTION}

The great advancement and wide application of modern science technology represented by communication technologies such as computer and communication has brought an omnidirectional and fundamental change to the military field, and at the same time, it has converted the army in the battle ground of the 21st century into the Digitalize Troops supported by information network which can achieve distributed commanding, linkage of essentials, self-harmonize, dynamic organizing, and share of information. The Digitalize Troop that our army is planning to build is based on the current Mechanized Troop, the extrusive characteristic of it is the addition of the building of information equipments and units, and information network is the ligament of the comprehensive network consists of every unit, distributed wireless network, tactical transmitter network, tactical satellite network and other networks. On the application level, it consists of nodes like command and control, reconnaissance and intelligence, firepower strike, and logistics.
A field-fixed combined way of constructing networks must be taken in the building of Digitalize Troop's information network, we should break the rigid traditional framework and make changes to the big, middle, small echelon situation, and the high, middle, low borderline in the use of military powers, combining the need and characteristics of Integrative Joint Operations to meet the demands of constructing a rationalized, comprehensive information network.

\section{THE BASIC CHARACTERISTICS OF DIGITALIZE TROOP'S INFORMATION NETWORK}

As to the Digitalize Troop that our army is planning to construct, many experts in military area have set high expectations for it. And for information network, it is required to be able to "transmit any information at any time anywhere" and link each node as completely as possible, that is to enable every node to communicate and share information with each other. This point of view roots in Metcalfe's law[1]: A complete-link network made up of $\mathrm{N}$ nodes, the quantity of the underlying information-link is $\mathrm{N}^{*}(\mathrm{~N}-1)$, when $\mathrm{N}$ is large enough, this value-law multiplies in index, that is to say, the value of a network multiplies with the square of the increase of the number of nodes. But in real world, when planning the structure of the Digitalize Troop's information network, whether the equipment node described before should be inserted into the network by complete connection or not, or rather there is a better way of organizing to fulfill its maximum potential needs a further analyze into the structure of the network.

The information network of Digitalize Troops itself is a complex network, different kinds of node units communicate with each other by means of fixed-connection and random-connection and in between. For example, in battlefield regional network, the connections between each main node are relatively stable, it belongs to the fixed-connection, while in actual combats, many other random elements exist, for example, the connection between wireless distributing network, tactical radio station network, and main networks belong to random-connection. Although 
the connection between all nodes differ in close and distant, the coexistence of this stable and random connections reflects the way of modeling the middle and small networks in complex network. And at the same time, the information network of Digital Troops should have the ability of high rate of information transmission and relatively good self-adjustment, self-synchronization, this exactly corresponds with the relatively short average route and high density of coefficient. So, using small-world model and relative measure to analyze the basic characteristics of information network can reflect some essence rules.

\section{THE CONSTRUCTION AND ANALYSIS OF DigitALIZE TROOP'S INFORMATION NETWORK MOde BASED ON COMPLEX NeTWORK}

The value of information network of Digitalize Troops is decided by the gain of a network, and the gain of network is normally made up of the income and loss of a network. The connection and congregation of a network embodies the ability of information sharing and efficiency of information transmission of a network, this is called the income of a network. But on the other hand, the complexity and cost it brings have become the spoilage of the network. So only by means of studying the network gain from both income and spoilage can we get the changing law of network value.

A. The construction of network models

1) The connection income of Digitalize Troop's information network $(\mathrm{I}(\mathrm{k}))$

The average length of the route of a network 1 represents the average distance between every two nodes, when $\overline{1} \rightarrow 0$, the connect gain of the whole network $\mathrm{I}(\overline{1}) \rightarrow 1$; when $\overline{1} \rightarrow \infty$, then $\mathrm{I}(\overline{1}) \rightarrow 0$;

When 1 decrease gradually, the connect gain of the whole network increases as well and tends to be 1 gradually, the gain function can be described as:

$$
\mathrm{I}(\overline{1})=\frac{1}{\omega \overline{1}+1}
$$

In it $\omega=\omega(p)$, meaning network information connecting element.

Using the knowledge of computer network, the average route length 1 of a complex network with small-world characteristic can be described as (The formula get by Newman and other people by means of revising and deplaning) [2]:

$$
l(N, k, p)=\frac{2 N}{k} f(N k p / 2)
$$

In it, $\mathrm{N}$ is the node of the network, $\mathrm{p}$ is the connection probability of the network, $\mathrm{f}(\mathrm{u})$ is a universal function, it satisfies:

$$
f(u)=\left\{\begin{array}{cc}
\text { cons } \tan t, & u<<1 \\
(\ln u) / u, & u>>1
\end{array}\right.
$$

Newman and other people gave the following approximation on the basis of Average Field:

$$
\mathrm{f}(\mathrm{x}) \approx \frac{1}{2 \sqrt{\mathrm{x}^{2}+2 \mathrm{x}}} \arctan \mathrm{h} \sqrt{\frac{\mathrm{x}}{\mathrm{x}+2}}
$$

The large amount of node units in the Digitalize Troops, because it can meet the requirements in formula (3) $u>>1$, so we can put $f(u)=(\ln u) / u$ into (1) and get:

$$
\mathrm{I}(\mathrm{N}, \mathrm{k}, \mathrm{p})=\frac{1}{\omega \overline{\mathrm{l}}+1}=\frac{1}{\omega \frac{4 \mathrm{~N}}{\mathrm{k}}(\ln (\mathrm{Nkp} / 2)) / \mathrm{Nkp}+1}=\frac{1}{\omega \frac{4 \ln (\mathrm{Nkp} / 2)}{\mathrm{k}^{2} \mathrm{p}}+1}
$$

Suppose $\alpha=4 \omega / p, \beta=N p / 2$, thus expression (5) can be simplified as:

$$
\mathrm{I}(\mathrm{k})=\frac{1}{\frac{\alpha \ln (\beta \mathrm{k})}{\mathrm{k}^{2}}+1}
$$

2) the wear cost of information network in digitize troops $(\mathrm{W}(\mathrm{k}))$

The average degree $\mathrm{k}$ of network represents the average connect level of nodes in the network, and with the increase of $\mathrm{k}$, connects of nodes in the network will gradually become over verbose leading to information overload, therefore increase the connect cost of the whole network, while reduce the gain of the network. When $\mathrm{k} \leq \mathrm{K} / 2$, $\mathrm{P}(\mathrm{k})=0$, and when $\mathrm{k} \geq \mathrm{K} / 2$, the arrangement of degree is[3]:

$\mathrm{P}(\mathrm{k})=\sum_{\mathrm{n}=0}^{\min (\mathrm{k}-\mathrm{K} / 2, \mathrm{~K} / 2)}\left(\begin{array}{c}\mathrm{K} / 2 \\ \mathrm{n}\end{array}\right)(1-\mathrm{p})^{\mathrm{n}} \mathrm{p}^{(\mathrm{K}-2)-\mathrm{n}} \frac{(\mathrm{pK} / 2)^{\mathrm{k}-(\mathrm{K} / 2)-\mathrm{n}}}{(\mathrm{k}-(\mathrm{K} / 2)-\mathrm{n}) !} \mathrm{e}^{-\mathrm{pk} / 2}$

Capture features of the equation, simplify it and find out that the wear function is:

$$
\mathrm{W}(\mathrm{k})=1-\mathrm{e}^{-\lambda \mathrm{k}}
$$

Thereinto $\lambda$ is the coefficient of wear cost in the network connect 
3) value gain of information network in digitize troops $(\mathrm{G}(\mathrm{k})$ )

By the token, as for information network with specific average degree $\mathrm{k}$, the value gain of the whole network is[4]:

$$
\begin{gathered}
\mathrm{G}(\mathrm{k})=\mathrm{I}(\mathrm{k})-\mathrm{W}(\mathrm{k}) \\
\mathrm{G}(\mathrm{k})=\frac{1}{\frac{\alpha \ln (\beta \mathrm{k})}{\mathrm{k}^{2}}+1}-\left(1-\mathrm{e}^{-\lambda \mathrm{k}}\right)
\end{gathered}
$$

\section{B. model value analysis}

According to formula (10), the value gain curve of the network can be reached, given all kind of information network nodes $\mathrm{N}=100$ existing in the marine corps include armaments like control vehicle, infantry fight vehicle, reconnaissance motor vehicle, nodal point communication vehicle and so on, and inter-nodal average connect probability $\mathrm{p}=0.01$, that is, the connect relationship among armaments is between complete regulated network and complete random network, and appeals more to a complete regulated one.(according to related knowledge from complex network theory, it can be found out that $\mathrm{p}=0$ refer to a complete regulated network , and $\mathrm{p}=1$ refer to complete random network). Draw curve illustrations as follows via Matlab software: follow:

Analyze the curve illustration, and reach conclusions as

$$
\text { 1). } \mathrm{G}(\mathrm{k}) \text { is a continuous function, }
$$

when $\mathrm{k} \rightarrow 0, \mathrm{G}(\mathrm{k}) \rightarrow 0$, when $\mathrm{k} \rightarrow \infty$, $\mathrm{G}(\mathrm{k}) \rightarrow 0$

, that is, with the increase of nodal degree, the value gain of network doesn't always increase, while it increase at the first place and it declines then, and it will not always be better with more connect among networks, but a most classic average degree.

2).although compared with normal troops, digitalize troops reach fairly high in the network-type degree, but if it is to set the information network to coupling network in complete oblate reticulation under entire situation, even though it can be realized in technical and financial support, the network value makes no gains, for that with the increase of network connect degree, its mass of verbose information not only reduces use efficiency, but also increase complexity of network' structure. Therefore, when going about design for structure of information network in digitalize troops later, and basing on keeping to capability of equipment, we are supposed to make inter-nodal connect meetas much

$$
\left.\frac{d G(k)}{d(k)} \rightarrow 0\right|_{k=k^{*}}
$$

as possible.

\section{EVOLUTION EXPERIMENT ANALYSIS}

In order to judge how rational the network gain is, this chapter use Netlogo ,a modeling tool, to design a evolutive experiment of network connect, also suppose that original network has 100 nodes in all, with on connections, that is ,with no information flows flowing in network, then margin the network randomly according to time length, to observe changes of network connections , and to search for the rational network connect level, i.e. the features of network when network gain reaches its maximum, and at last build specific algorithm compiled by Netlogo, of which the main codes are as follows;

to go

;; When conditions below are met, we will get a complete connected network, and it stops growing

if $((2 *$ count links $)>=($ (count turtles) $*$ (count turtles - 1)) ) [

display

user-message "Network is fully connected. No more edges can be added."

]

$$
\begin{aligned}
& \text { stop } \\
& \text { ] add-edge } \\
& \text { find-all-con } \\
& \text { color-giant-co } \\
& \text { ask links [ } \\
& \text { tick } \\
& \text { do-plotting } \\
& \text { layout }
\end{aligned}
$$

find-all-components

color-giant-component

ask links [ set color [color] of end1 ]

end

amid the growing process of network:

; Search for all the components in the network, set nodes with attributes, and make it search of itself

ask turtles [ set explored? false ]

;; Keep growing until all nodes are found loop

already

[ ; search for nodes which have not been found

let start one-of turtles with [ not explored? ]

if start $=$ nobody [ stop ]

;; reset the number of nodes to 0

\#; the variable updates every time when a new node is found

$$
\text { set component-size } 0
$$$$
\text { ask start [ explore (gray }+2) \text { ] }
$$
component

; update the variable referring to the size of the

;; inspect whether there are new ingredients

if component-size $>$ giant-component-size

[ set giant-component-size component-size

end

$$
\text { set giant-start-node start ] ] }
$$

operations aimed at sides:

;; search for a lot margin randomly and rebuilt it

to add-edge

let node1 one-of turtles

let node 2 one-of turtles

ask node1 [

ifelse link-neighbor? node 2 or node $1=$ node 2

;; if there has been a side already, return it

; search for new nodes again 
[ add-edge ]

$; ;$ if not, rebuild it

[ create-link-with node2 ] ]

In order to describe the evolution of network, we use the 2 illustrations below to represent its process. A represents the variation of the link of network during its process varying from 0 to 100 ; $\mathrm{B}$ represents the variation of the average length of network when proportion of side and point is over 3

In combination with the knowledge from complex network, analyzing the illustrations can draw solutions as follows:

1. The growth of network has a critical state, when the linking sides is to reach the number of nodes, it will form a connected network rapidly.

2. When the number of sides is almost equal to that of its nodes, the most connected network is nearly to reach its limit-90. Then, the size of network grows more slowly and when the proportion reaches $2: 1$, the network is completely connected, and 100 isolated nodes can form a connected network.

3. As the number of sides further increases, and when the proportion is 3, it's time to review its average length. The average length will endure a exponential decrease with the growth of side, first it will go up as the side's number grows, the average length formed by points decreases quickly, and the curve turns to be precipitous. But when the number of side is $500-600$, it decreases not that fast, the curve turns to be smooth. That is to say, when the number of side is 5 to 6 times of that of point, the effect caused by number of side to average length lower, and so does its speed. Seeing from the benefit of network, i.e. after the proportion of connect level is $6: 1$, the value gain of network tends to its maximum. Later, its complicacy will go up and the growth of value gain is not that obvious.

4. In order to guarantee that connect value in information network reaches its maximum, when constructing the structure of information networks, the rational network connect level, i.e. the proportion between sides and nodes should be kept within 2 6, if it is less than 2, its features of network won't be fully formed, with some node units not connected, but if it is more than 6 , the value gain grow slowly, and at the same time because of a series of complexity brought by the increase of the side numbers, it thus will reduce structural running efficiency.

5. From the critical state formed by explosive growth seen from network evolution research, nearly $90 \%$ growth of network is done by around $10 \%$ growth of side's number, this is a feature which should be fully made use of during the process of building up a information network in digitalize troops. An important phenomenon in the evolution of network is the growth of accidents, which also means when a small group of nodes form a bigger and more complex network, its connectivity will suddenly grows. When the digital troops are dealing with the joint operation, its information network should be a dynamic structure, which means a potential structure which can make judgments based on variation of the battlefield and can also maintain the connectivity, called "potential structure" [5]. It can be seen as a dynamic network connecting well in parts but can maintain doubtful in the whole part to make enemies confused , making the enemies' exact strike against our "important nodes" fail. At the meantime, the digitalize troops can quickly match the network at a suitable time and spot, and make it back to a fuzzy state after military operations. The exist of potential structure can also contribute to the smooth transformation of network, when the environment and task changes in substance, the deployment of links and nodes may can not match with the new environment and task, the network needs to be rearranged to adapt for the new aims. Because of the potential structure, only $10 \%$ links need deployed again and this can reconstruct the network dynamically. This property is also very important. According to the knowledge of complex network, the potential network bear fruits only when forming a network dynamically or rearranged, we can measure the ability of network to suit it automatically and reconstruct dynamically via analyzing the number of potential structures.

\section{SUMmary}

Though the theory raises a very strict request to the construction of digital troops, and also sees the completed connectivity as a hope for the high transportation of information and the self-defense of network, but this request is only centered on the pro side of high connectivity and the number of points on lifting the benefit of network, but ignores the complexity caused by its cost and harm to informational network. And seeing from the structure of network, the informational network of digital troops in the future should not be a completely connected network by all the points, but a suitably connected, reasonably flat and dynamically modular structure.

\section{ACKNOWLEDGMENT}

This work is partially supported by NSF Grant \#70771112 and the NCET-06-0936.

\section{REFERENCES}

[1] Alberts. Network Centric Warfare: Developing and Leveraging Information Superiority (2ndEdition). CCRP Serials Publication, 2000

[2] Moffat. Complexity Theory and Network Centric Warfare. CCRP Serials Publication, 2000

[3] Watts D J. Small Worlds: The Dynamics of Networks between Order and Randomness. Princeton, New Jersey: Princeton University Press, 1999

[4] Duolin Zhang. Estimate on the Efficiency of Air-Defense in Informational Warfare Based on Fuzzy Comprehensive Reviewing. 2003(5)

[5] Jeffrey R. Cares. An Information Age Combat Model. 9th International Command and Control Research and Technology Symposium, 2004 


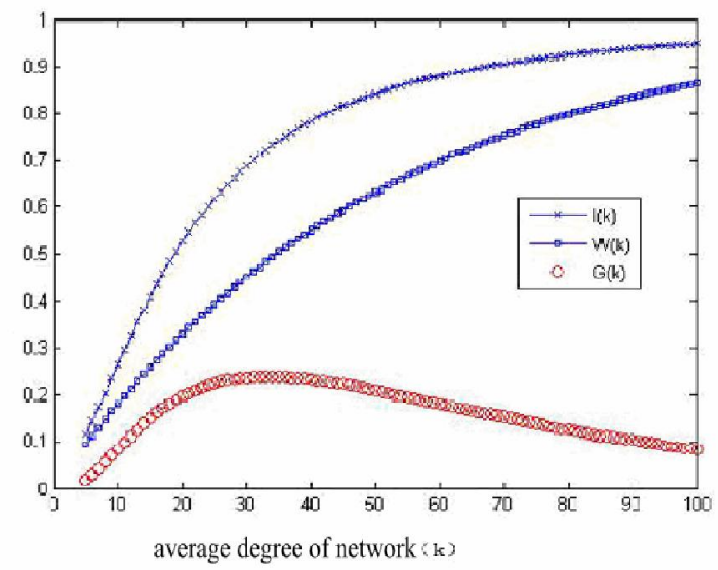

Figure.1 curve of variation of information network gain based on average nodal degree changes

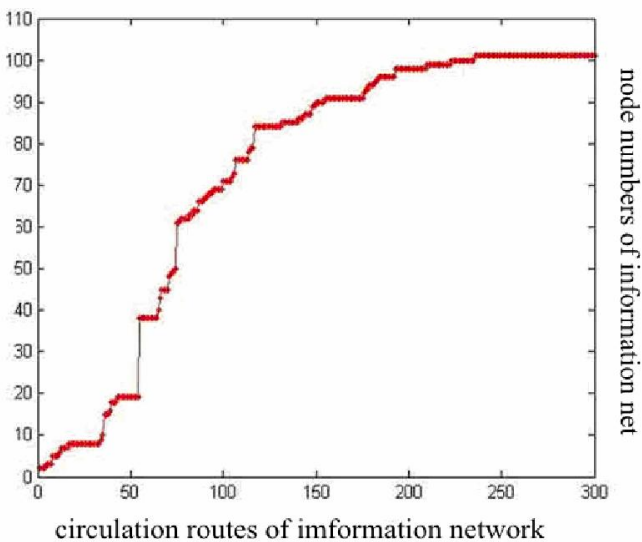

Figure. 2 A

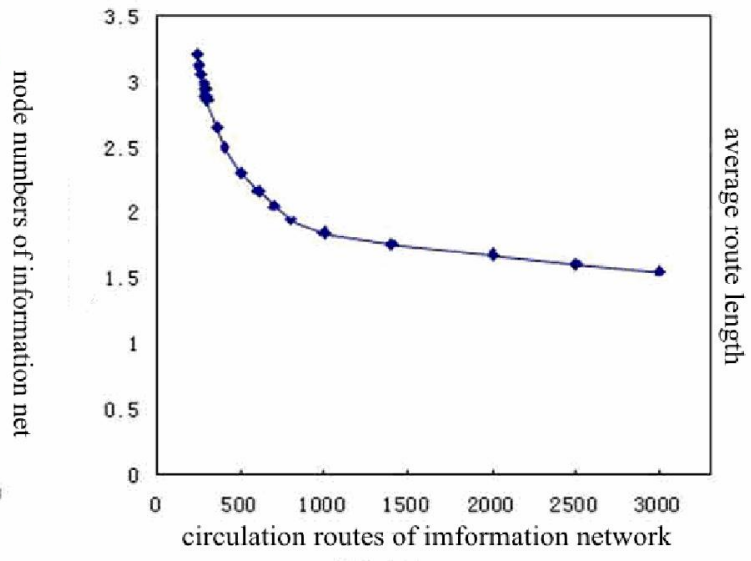

B

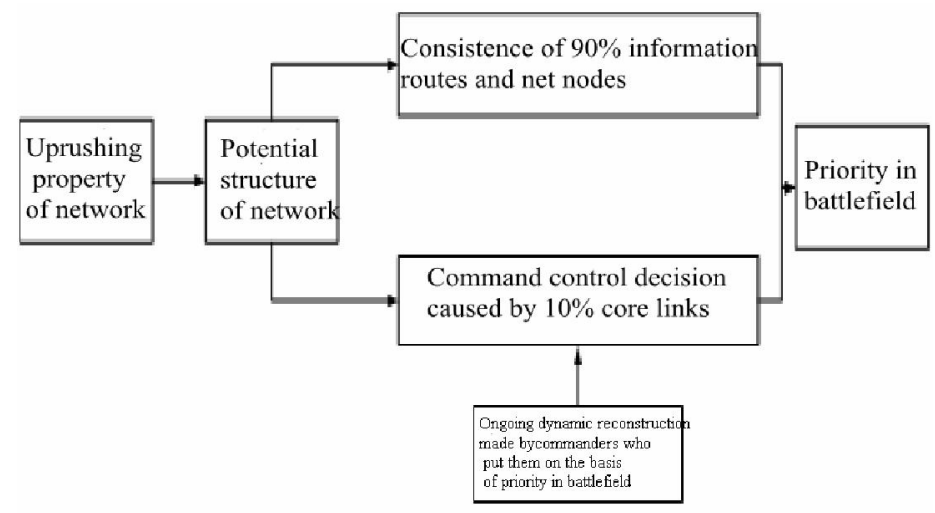

Figure 3. sketch map for exertion of network evolution growth 